\title{
ON A METHOD OF COMPARISON FOR STRAIGHT-LINE NETS*
}

\author{
BY LOUISE D. CUMMINGS
}

1. Introduction. The problem which led to this investigation of the structure of systems of straight lines in a plane was undertaken for the purpose of reclassifying, if possible, the 36 Weber-Aronhold sets of seven real double-tangents of a plane quartic curve. Different ways of constructing straight-line nets are known, but easy and conclusive methods for comparing systems apparently different are at least not well known.

We exhibit here a new method of comparison for straightline nets and apply it to show all non-equivalent types for $n=6,7$, and all heptagonal types for $n=8$.

2. Non-Equivalent Sets of $n$ Lines. A set of real lines, finite in number, in the plane of projective geometry, divides the plane into convex polygons. If no three of the lines meet in a point, then by Euler's equation $n$ lines form $\left(n^{2}-n+2\right) / 2$ polygons. Two sets of $n$ lines are equivalent when a one-to-one relation exists between the lines and polygons of the two sets. If $n<6$, the sets are equivalent, but for $n \geqq 6$, four or more non-equivalent sets are known to exist. $\dagger$

3. Method of Construction. Since all sets of five lines are equivalent, any five lines in a set may be selected as the initial five. Adding a sixth line, we construct systems of six lines whose equivalence or non-equivalence is tested by this new method of comparison, and only four different types exist. Employing these four types of six lines as initial sets and adding a seventh line we find that only eleven non-equivalent sets of seven lines exist. Among the eleven non-equivalent sets of seven lines, one set contains a convex heptagon. From this set by adding an eighth line we derive fifteen non-equivalent sets of eight lines, designated as heptagonal sets of eight lines.

* Presented to the International Congress of Mathematicians, Zurich, September 5, 1932.

$\dagger$ H. S. White, this Bulletin, vol. 38 (1932), p. 59; and L. D. Cummings, this Bulletin, vol. 38 (1932), p. 105; also vol. 38 (1932), p. 700. 
4. Notation and Method of Comparison. Let $l_{1}$ and $l_{2}$, copunctual at $p$, be any two of a net of $n$ lines. On these two lines lie $(2 n-3)$ of the $n(n-1) / 2$ points of intersection of the net. The two lines divide the plane into two regions I and II inside of which the remaining $(n-2)(n-3) / 2$ points of intersection lie.

The index of the point $p$ shows the exact distribution in regions I and II of these residual points of intersection. The $n(n-1) / 2$ indices of all the points of intersection of the $n$ lines are determined, like indices are collected together, and the tabulated result is designated as the index of the system. The index of any system is a unique characterization of that system and furnishes probably a sufficient test for the equivalence or non-equivalence of that system with any other system.

5. Systems of Six Lines. For brevity this new method of comparison is shown in its application to systems of six lines. Five

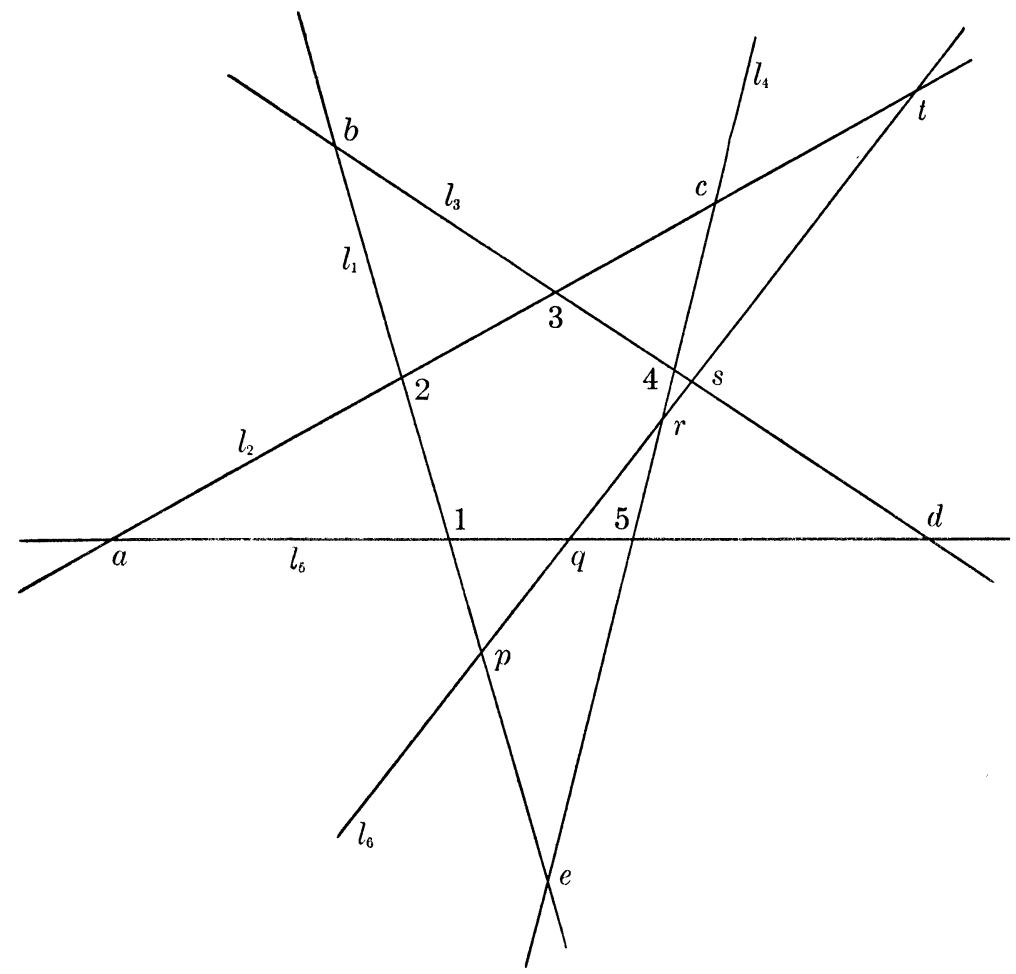

Fig. 1. System (1). 
lines divide the plane into eleven convex polygons, one pentagon, five triangles, and five quadrilaterals. A pentagon with sides produced indefinitely and all ten intersections in the finite plane is selected as typical arrangement for $n=5$. Let there be given a sixth line or secant crossing (i) two consecutive sides of the pentagon as in Figure 1, System (1).

Designate the vertices of the pentagon in order $1,2,3,4,5$; the vertices of the five triangles in the same cyclic order $a, b, c$, $d, e$; and the intersections of the sixth line $l_{6}$ with the sides of the pentagon $p, q, r, s, t$. Let the sides $12,23,34,45,51$ of the pentagon be $l_{1}, l_{2}, l_{3}, l_{4}, l_{5}$.

For $n=6$, we have $n(n-1) / 2=15,(2 n-3)=9$, and there are $(n-2)(n-3) / 2=6$ residual points. At point 1 the two lines $l_{1}$ and $l_{5}$ divide the plane into two regions I and II. Region I contains none of the residual points of intersection and region II contains six of them, and point 1 has the $i$ dex $(0,6)$, written 1 $(0,6)$; the point $d$ has index $(2,4)$ or $d(2,4) ; p(3,3)$; and this system has no points with index $(1,5)$. The index $(i, j)$ of any point is always written with the smaller number first and may be abbreviated to $i$ as in the column-headings of Table III.

Figure 1, System (1) contains six points 1, 2, 3, 4, r, $q$ with index $(0,6)$; no points with index $(1,5)$; three points $d, e, t$ with index $(2,4)$ and six points $a, b, c, s, 5, p$ with index $(3,3)$. These results are given in tabular form as

\begin{tabular}{ccccc} 
System & \multicolumn{4}{c}{ IndicES } \\
& $(0,6)$ & $(1,5)$ & $(2,4)$ & $(3,3)$ \\
\hline$(1)$ & 6 & 0 & 3 & 6
\end{tabular}

The group of order 12 generated by

$$
\begin{aligned}
& S_{1} \equiv(d e t)(1234 r q)(a b c s 5 p) \\
& S_{2} \equiv(13)(a b)(c p)(d)(e t)(4 q)(5 s)(2)(r)
\end{aligned}
$$

transforms the system (1) into itself. This method of indices simplifies the determination of all substitutions which transform a system into itself or into any equivalent system.

The investigation of all possible positions for the sixth line or secant shows only three additional non-equivalent systems. In the following Table I the indices for each of the four non-equivalent systems are given and also, incidentally, the actual polyg- 
TABLE I

The Plane Figure of Six Real Lines

\begin{tabular}{l|cccc|ccccc}
\hline \hline \multirow{2}{*}{ System } & \multicolumn{5}{|c|}{ Indices } & \multicolumn{5}{|c}{ Polygons } \\
\cline { 2 - 8 } & $(0,6)$ & $(1,5)$ & $(2,4)$ & $(3,3)$ & $S_{6}$ & $S_{5}$ & $S_{4}$ & $S_{3}$ \\
\hline$(1)$ & 6 & 0 & 3 & 6 & 1 & 0 & 9 & 6 \\
$(2)$ & 4 & 4 & 4 & 3 & 0 & 2 & 8 & 6 \\
$(3)$ & 3 & 6 & 6 & 0 & 0 & 3 & 6 & 7 \\
$(4)$ & 0 & 15 & 0 & 0 & 0 & 6 & 0 & 10 \\
\hline
\end{tabular}

onal division of the projective plane, satisfying of course the Euler equation

$$
s_{6}+s_{5}+s_{4}+s_{3}=\frac{n^{2}-n+2}{2},
$$

where $s_{i}$, the number of polygons of $i$ sides, is tabulated for each of the four systems.

6. Systems of Seven Lines. To form the systems of seven lines, each of the four non-equivalent systems of six lines of

TABLE II

The Plane Figure of Seven Real Lines

\begin{tabular}{|c|c|c|c|c|c|c|c|c|c|c|c|}
\hline \multirow{2}{*}{ System } & \multicolumn{6}{|c|}{ INDICES } & \multicolumn{5}{|c|}{ Polygons } \\
\hline & \multicolumn{6}{|c|}{$(0,10)(1,9)(2,8)(3,7)(4,6)(5,5)$} & $S_{7}$ & $S_{6}$ & $S_{5}$ & $S_{4}$ & $S_{3}$ \\
\hline (1) & 7 & 0 & 0 & 0 & 14 & 0 & 1 & 0 & 0 & 14 & 7 \\
\hline (7) & 1 & 6 & 6 & 4 & 2 & 2 & 0 & 1 & 5 & 5 & 11 \\
\hline (8) & 3 & 0 & 12 & 0 & 6 & 0 & 0 & 1 & 3 & 9 & 9 \\
\hline (3) & 4 & 2 & 3 & 6 & 4 & 2 & 0 & 1 & 2 & 11 & 8 \\
\hline (2) & 5 & 2 & 0 & 6 & 4 & 4 & 0 & 1 & 1 & 13 & 7 \\
\hline (11) & 0 & 9 & 3 & 3 & 6 & 0 & 0 & 0 & 6 & 6 & 10 \\
\hline (5) & 2 & 6 & 3 & 2 & 6 & 2 & 0 & 0 & 5 & 8 & 9 \\
\hline (6) & 3 & 4 & 4 & 2 & 2 & 6 & 0 & 0 & 4 & 10 & 8 \\
\hline (10) & 2 & 4 & 5 & 6 & 2 & 2 & 0 & 0 & 4 & 10 & 8 \\
\hline (4) & 4 & 2 & 3 & 4 & 8 & 0 & 0 & 0 & 3 & 12 & 7 \\
\hline (9) & 3 & 3 & 6 & 0 & 6 & 3 & 0 & 0 & 3 & 12 & 7 \\
\hline
\end{tabular}

When, in a set of seven lines, any six form a convex hexagon, the system is designated as hexagonal.

The eight systems (1), (2), (3), (4), (5), (6), (7), (8) are hexagonal and the three systems (9), (10), (11) are non-hexagonal. 
Table I is used as a basic system and all possible positions for the seventh line or secant are investigated.

Table II exhibits the indices for each of the eleven nonequivalent systems of seven real lines in the plane, and shows also the polygonal division of the plane for each of these systems. If in a system of seven lines any six form a convex hexagon, the system is designated as an hexagonal system of seven lines. In Table II the eight systems (1), (2), (3), (4), (5), $(6),(7),(8)$ are hexagonal systems derived from the basic system (1) of Table I. When systems (2), (3), (4) of Table I are used as basic systems only three additional non-equivalent systems (9), (10), (11) of Table II are obtained and these are designated the pentagonal systems of seven lines.

Systems (6) and (10) in Table II have different indices but the same invariant numbers in the polygonal divisions, and show that equality of polygonal numbers is a necessary but not a sufficient condition for the equivalence of two systems. A comparison of systems (4) and (9) shows the same fact.

7. Systems of Eight Lines. To determine the non-equivalent systems of eight lines I assume the eleven non-equivalent systems of Table II as bases and examine all possible positions of the eighth line. A system of eight lines is designated as heptagonal if any seven of the lines form a convex heptagon. Table III shows the indices and the polygonal numbers for each of the fifteen non-equivalent heptagonal systems of eight lines derived from the base system (1) of Table II. In Table III the three non-equivalent systems (15), (12), and (14) have the same invariant polygonal numbers but quite different indices.

8. Conclusion. We have now two distinct methods of comparison: that of marks developed by $\mathrm{H}$. S. White* and this of indices, both easy of application, which have given reliable and accordant results, positive as well as negative, in all cases in which they have been employed. Both methods lead to the easy discovery of the substitutions which transform any system in to itself or into an equivalent system. The two methods have been applied to 36 Weber-Aronhold sevens and show a new distribution of the same into 11 non-equivalent subdivisions.

* The plane figure of seven real lines, this Bulletin, vol. 38 (1932), pp. 59-65. 
TABLE III

Heptagonal Systems of Eight Lines in a Plane

\begin{tabular}{|c|c|c|c|c|c|c|c|c|c|c|c|c|c|c|}
\hline \multirow{2}{*}{ System } & \multicolumn{8}{|c|}{ INDEX } & \multicolumn{6}{|c|}{ Polygons } \\
\hline & 0 & 1 & 2 & 3 & 4 & 5 & 6 & 7 & $S_{8}$ & $S_{7}$ & $S_{6}$ & $S_{5}$ & $S_{4}$ & $S_{3}$ \\
\hline (1) & 8 & 0 & 0 & 0 & 0 & 8 & 4 & 8 & 1 & 0 & 0 & 0 & 20 & 8 \\
\hline (11) & 0 & 7 & 0 & 7 & 7 & 0 & 0 & 7 & 0 & 1 & 0 & 7 & 7 & 14 \\
\hline (15) & 2 & 6 & 0 & 3 & 3 & 5 & 7 & 2 & 0 & 1 & 0 & 5 & 11 & 12 \\
\hline (12) & 2 & 3 & 4 & 2 & 8 & 2 & 2 & 5 & 0 & 1 & 0 & 5 & 11 & 12 \\
\hline (14) & 2 & 4 & 4 & 0 & 6 & 4 & 6 & 2 & 0 & 1 & 0 & 5 & 11 & 12 \\
\hline (13) & 4 & 0 & 4 & 3 & 5 & 6 & 0 & 6 & 0 & 1 & 0 & 3 & 15 & 10 \\
\hline (3) & 5 & 2 & 1 & 1 & 3 & 5 & 8 & 3 & 0 & 1 & 0 & 2 & 17 & 9 \\
\hline (4) & 5 & 1 & 2 & 2 & 2 & 6 & 8 & 2 & 0 & 1 & 0 & 2 & 17 & 9 \\
\hline (2) & 6 & 2 & 0 & 0 & 2 & 6 & 7 & 5 & 0 & 1 & 0 & 1 & 19 & 8 \\
\hline (6) & 2 & 5 & 3 & 0 & 5 & 2 & 6 & 5 & 0 & 0 & 1 & 5 & 12 & 11 \\
\hline (7) & 3 & 4 & 1 & 3 & 3 & 3 & 7 & 4 & 0 & 0 & 1 & 4 & 14 & 10 \\
\hline (5) & 3 & 4 & 2 & 1 & 4 & 3 & 6 & 5 & 0 & 0 & 1 & 4 & 14 & 10 \\
\hline (9) & 4 & 1 & 4 & 2 & 2 & 6 & 2 & 7 & 0 & 0 & 1 & 3 & 16 & 9 \\
\hline (10) & 4 & 4 & 0 & 1 & 4 & 3 & 6 & 6 & 0 & 0 & 1 & 3 & 16 & 9 \\
\hline (8) & 5 & 1 & 1 & 3 & 3 & 5 & 4 & 6 & 0 & 0 & 1 & 2 & 18 & 8 \\
\hline
\end{tabular}

A system of 8 lines is heptagonal if any seven of the lines form a convex heptagon.

The intrinsic difference between Weber's basis of classification of sets of seven lines and that investigated in this paper is that Weber relates the heptads to a quartic curve and this paper studies them as sets of lines, without relation to any other figure.

This method of analysis of straight-line nets by the indices, as herein extended to all the points of intersection of the system, regardless of their relation to the vertices of a pentagon, hexagon, or other basal polygon, is applicable to any number $n$ of straight lines and moreover is not restricted to the case that only two straight lines pass through a point.

Vassar College 